\title{
Kinetic Analysis on the Motility of Liverwort Sperms Using a Microscopic Computer-Assisted Sperm Analyzing System
}

\author{
Takuya FuRUiCHI $^{1}$ and Koji MATSUURA ${ }^{2}$ \\ 'Department of Human Life Sciences, Nagoya University of Economics, Inuyama, Aichi 484-8504, Japan \\ ${ }^{2}$ Cardiovascular Physiology, Graduate School of Medicine, Dentistry and Pharmaceutical Sciences, \\ Okayama University, Okayama 700-8558, Japan
}

(Received September 1, 2015; Accepted November 9, 2015)

\begin{abstract}
Some of the plant species including embryophytes (especially, bryophytes and ferns) utilize the sperms but not pollens for their sexual reproduction. Because sperm motility is one of the most important features for the fertility in human and animals, percentage of motility and the kinetic parameters for swimming and the guidance to eggs (taxis) are well focused in the field of reproductive biology to evaluate and improve the male infertility. However, the nature of plant sperms is rarely known even more than a century has passed since the first microscopic observations of sperms in embryophytes as in the cases in Ginkgo biloba and Cycas revoluta, commonly known as sperm-generating plant species, have been made in the end of 19th century. In the present study, we performed high throughput analysis on the sperm motility in the most common liverwort, Marchantia polymorpha L. using a Computer-Assisted Sperm Analyzing (CASA) system. Time-dependent changes in kinetic parameters of Marchantia sperm motility from high to low motility states suggested that amplitude of lateral head displacement, rather than its frequency play key roles in the speed and distance of swimming at high motility state. From the average lifetime of high motility state and the speed of migration, the distance traveled by Marchantia sperms, due to their own motility, are estimated to be less than $3 \mathrm{~cm}$, suggesting that motility of Marchantia sperms might mainly play a key role in the final fertilization step, but not in the long-distance travelling from the patchily distributed colonies of male strains to those of female strains, often being apart by few meters in the field.
\end{abstract}

Keywords : Marchantia polymorpha L., motility, sperm

\section{INTRODUCTION}

Embryophytes (bryophytes and ferns) perform both parthenogenesis and sexual reproduction in their life cycle, and sperms are used for their sexual reproduction, in place of pollens in higher plants (Renzaglia et al., 2000; Renzaglia and Garbary, 2001). Because sperm motility is one of the most important features for the fertility in human and animals, percentage of motility and the kinetic parameters for swimming and the guidance to eggs (known as taxis) are well focused in the field of reproductive biology to evaluate and improve the male infertility. However, the nature of plant sperms is rarely known even more than centuries has passed since the first description on the discharge of samen from embryophytes by Schmidel in 1747 (as note by Mirbel, 1835a; 1835b; Thuret, 1851; Cavers, 1903; 1904; see also recent review by Araki, 2012). The discovery of a freely swimming sperm in Ginkgo biloba at the end of the nineteenth century by Sakugoro Hirase was one of the most significant moments in botany (Ridge et al., 1997). In the end of nineteenth century, the freely swimming sperms in embryophytes, thus motile under microscopes, were first identified in Ginkgo biloba (Hirase, 1896a; 1896b) and Cycas revoluta (Ikeno, 1896; 1897; Ikeno and Hirase, 1897), nowadays known as members of sperm-generating plant species.

As the first step in fertilization mechanism in bryophytes, semen enriched with the biflagellated sperms are discharged from the antheridia of males as the top of antheridial receptacle are wet by the rain drops (Carothers and Kreitner, 1967; 1968, Shimomura, 2012). Discharge of semen is promoted by a droplet of water but not by touch, indicating that swelling rather than mechanical stimuli, and some following signal transduction might be playing key role(s) in the process. For the opening of covering jacket of an antheridium and distending of the antheridium wall cells which give a pressure to discharge sperms, swelling of the cells and mucus are supposed to be important (Renzaglia et al., 2000; Shimomura, 2012), which is different from the motive contraction of alveoli of human breast for lactation, which initiated by baby's suck, a mechanical stress. A recent study on the "airborne" sperms of $C$. conicum indicated that liverwort sperms are discharged as pre-motile form, then shift to the motile form when they swell or they are subjected to hypo-osmotic stress (Shimomura et al., 2008). Thus, swelling and/or hypoosmotic stress, but not mechanical stress may distinctly play key roles in the phases of sperm discharge and transition to motile form in liverwort sperms.

In general, swimming ability is one of the most important features of sperms because they must reach and fertil-

Corresponding author: Takuya Furuichi, fax: + 81-568-67-4299, e-mail : furuichi@nagoya-ku.ac.jp 
ize the eggs for sexual reproduction. Thus, sperm motility analysis is the gold standard for investigating the cause of male infertility in the field of reproductive medicine. To investigate and evaluate the male fertility, ComputerAssisted Sperm Analyzing (CASA) system has been developed in recent decades and utilized as a representative method to evaluate male fertility, and thus, used as a conventional tool to obtain the kinetic parameters of sperm motility in the biological research fields (Komori et al., 2006; Matsuura et al., 2011; Amann and Waberski, 2014).

In case of plant sperms, roles or modes of swimming ability for the delivery of genetic information to the eggs are in arguments. Colonies of male and female strains are distributed in a non-uniform or patchy manner, sometimes male and female are positioned meters apart, but they successfully fertilize in the field. Meter-long distance might be beyond their swimming ability, thus water flow covering the field, air flow for airborne sperms, and insect pollination has been proposed as alternative delivery systems (Shimomura et al., 2008; Shimomura, 2012). Nevertheless, swimming ability of plant sperms should play an inevitable role in invasion into the oviduct of archegonium to achieve fertilization of the eggs. Thus, clarification of the motility and its kinetic parameters seems to be important for our understanding of the sexual reproduction in certain embryophytes and sperm-generating plant species.

Among the number of liverworts, Marchantia polymorpha $\mathrm{L}$. has been used as a model plants in recent studies by the establishment of genome-wide analysis and the conventional transformation techniques (Ishizaki et al., 2008). In the present study, we characterized the motility of Marchantia sperms using a CASA system, and discussed the role of their swimming ability for the sexual reproduction of liverworts.

\section{MATERIALS AND METHODS}

\section{Plant materials}

Male gametophyte of Marchantia polymorpha, accession Takaragaike-1 (Tak-1), was cultured at $22^{\circ} \mathrm{C}$ on moistened vermiculite under continuous light at $40-50 \mu \mathrm{mol}$ photons $\mathrm{m}^{-2} \mathrm{~s}^{-1}$ in a plant growth chamber (M-260F, TAITEC, Saitama, Japan). A conventional light illuminating panel of light emitting diodes (LEDs) (Lien Inc., Mie, Japan) designed for standard plant growth and sexual organ development (red : blue : far-red $=165: 50: 10$ ) was manually modified by replacing far-red light $(735 \mathrm{~nm})$ emitting LEDs (L735-01AU, Epitex, Kyoto, Japan) with additional 10 chips of blue light $(460-470 \mathrm{~nm})$ emitting LEDs, to promote the development of the sexual organ, and used as a light source for plant growth.

\section{Analysis of sperm motility}

Discharge of Marchantia semen was promoted by gentle loading of a droplet $\left(40^{-} 50 \mu \mathrm{L}\right)$ of double-distilled water $\left(\mathrm{ddH}_{2} \mathrm{O}\right)$ onto an antheridial receptacle under a stereomicroscope. The semen enriched with sperms, discharged from several antheridia on an antheridial receptacle, was immediately picked up by a micropipette. Because Marchantia sperms were adhesive to the hydrophilic surface such as of glass and eventually bound onto them as observed in porcine sperms (Matsuura et al., 2011), Marchantia semen were placed into $1.5 \mathrm{~mL}$ centrifuge tubes (siliconized) and stored at $22^{\circ} \mathrm{C}$ until used for measurements. For the analysis of sperm motility, an adhesive, punch hole reinforcement film (thickness, $0.05 \mathrm{~mm}$; hole diameter, $6 \mathrm{~mm}$; PSM10B, Kokuyo, Osaka, Japan) was attached onto a slide glass, and a $1-\mu \mathrm{L}$ aliquot was spotted onto the "well" on the slide glass (Matsunami Glass, Osaka, Japan) prior to be mounted with a cover glass. The depth of liquid pool on the slide was defined by the thickness of punch hole reinforcement film placed as the spacer between the slide glass and the cover slip.

A CASA system, designated as SMAS (Sperm Motility Analysis System (Ditect Co., Tokyo, Japan), consists of a high-resolution digital CCD camera and a computer with analyzing software was used for motility analysis of the Marchantia sperms. By the analyzing software, each image frame processed after image thresholding protocol is captured and the blight areas with appropriate size are recognized as heads of sperms, and the tracks of sperm's movement were recorded (Fig. 1a). Although the head parts of Marchantia sperms are not oval like human's sperm, thicker portion within the slender head shape with the oppositely positioned origins of a pair of flagella on the head (Fig. 1b), were successfully detected as a blight area resembling the head of human sperms, and the above imaging feature enabled us to analyze the motility of Marchantia sperms using SMAS.

Through a high-resolution CCD camera, SMAS tracks the sperm's movement and records the position of each

a

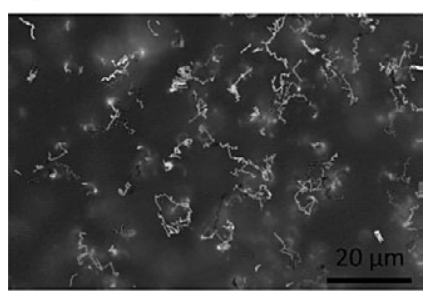

b

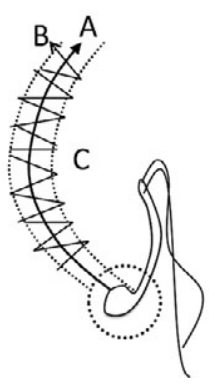

Fig. 1 CASA analysis of Marchantia sperm motility. a: Representative image of the tracks of sperm's movement for $1 \mathrm{~s}$ (30 min after discharge). b: Schematic diagram of the motility analysis of Marchantia sperm. Dotted circle indicate thicker, plastid containing part of head, where detected by SMAS. A: linear velocity, B: curvilinear velocity, C: amplitude of head place displacement (width of two dotted lines). 


\section{MOTILITY OF LIVERWORT SPERMS}

sperm through the negative-phase contrast imaging for $1 \mathrm{~s}$ at 60 frames s$^{-1}$. Because the sperms moved vertically as well as horizontally, some of the sperms went out from the focused plane during the recording (relatively short traces in Fig. 1a). Thus, statistic analysis for the kinetic parameters of the motion was performed with the tracks of sperms entirely recorded for $1 \mathrm{~s}$. With a negative phase-contrast microscopy, the system records the motion of individual sperms for $1 \mathrm{~s}$, to yield five parameters of motion; namely, (1) percentage of motility, (2) curvilinear and (3) linear (straight-line) velocity, (4) amplitude and (5) frequency of lateral head displacement. The data for each parameters of motion were analyzed with Excel 2004 (Microsoft Co., USA) and Igor Pro 5.0 (WaveMetrics, Lake Oswego, OR, USA). Sperms were collected from more than 20 antheridial receptacles of independent male strains, and the motility of $>2000$ sperms were analyzed. To estimate the mean values, the frequency (number of sperms per section) distribution histograms for each parameters and the Gaussian fitting were processed (Fig. 2).

\section{RESULTS AND DISCUSSION}

When the sperms were observed immediately after the discharge, all of them exhibited ring-like, pre-motile form, then most of them started rotation within $2-5$ min after discharge. Sperms started free swimming within 5-10 min after discharge, and then they enter the actively motile phase lasting for around $30 \mathrm{~min}$ after discharge. Histogram analysis for curvilinear velocity (total distance of swimming per unit of time) of swimming sperms in semen at 30 min after discharge revealed that the population of sperms could be divided into two apparent groups with high and low motilities (Fig. 2a). Gaussian fitting for each peaks estimated the mean peak amplitudes of high and low motility groups to be $58.93 \pm 0.39 \mu \mathrm{m} \mathrm{s}^{-1}$ and $22.73 \pm 0.38 \mu \mathrm{m} \mathrm{s}^{-1}$, respectively, and the ratio of highly active population over the less active population was 2.27. High motility population was diminished in $60 \mathrm{~min}$, and then low motility population became the dominant.

In contrast, the histogram for the frequency of lateral head displacement, mainly reflecting the rotation speed of flagella, showed almost symmetrical single peak at both 30 min and $60 \mathrm{~min}$ after discharge (Fig. 2d and e), and the mean values was not altered with time (30 min; 17.018 $0.229 \mathrm{~Hz}, 60 \mathrm{~min} ; 18.442 \pm 0.27 \mathrm{~Hz}$ ). It is tentative to speculate that the rotation angle, rather than speed of flagella could be one of the key features of high motility group. As the population of motile sperms was gradually lowered (30 min: 73\%, $60 \mathrm{~min}: 74 \%, 120 \mathrm{~min}: 38 \%$ ), average lifetime of motile Marchantia sperms ranges ca. 70-90 min after discharge under wet condition.

Histograms of the rest of parameters, linear (straightline) velocity and amplitude (width) of lateral head displacement showed broader distribution patterns without dissection of the population (Fig. $2 f$ and $j$ ). Thus, the sperms were separated into two distinct populations with high or low motility by their curvilinear velocity, and reanalyzed (Fig. 2g: $14.582 \pm 0.408 \mu \mathrm{m} \mathrm{s}^{-1}$, Fig. 2h: 5.3025 $\pm 0.187 \mu \mathrm{m} \mathrm{s}^{-1}$, Fig. $2 \mathrm{k}: 2.393 \pm 0.0471 \mu \mathrm{m}$, Fig. 21: $0.76081 \pm 0.0223 \mu \mathrm{m})$.

Mean values of linear velocity and amplitude of lateral head displacement of low motility population were also similar to that of sperms at $60 \mathrm{~min}$ after discharge (Fig. 2i: $4.47 \pm 0.395 \mu \mathrm{m} \mathrm{s}^{-1}$, Fig. $\left.2 \mathrm{~m}: 0.756 \pm 0.0591 \mu \mathrm{m}\right)$. It is further indicating that the amplitude, rather than the frequency of lateral head displacement is a key parameter representing the high motility.

All of kinetic parameters for sperm motility in Marchantia at $60 \mathrm{~min}$ after the discharge were at the range similar to the low motility population at $30 \mathrm{~min}$ after the discharge, suggesting that motility of Marchantia sperms time-dependently shifted from high to low motility states. In addition, rate of motile sperm has been drastically reduced by $120 \mathrm{~min}$. According to the linear velocity and the life of high motility state in the present results (ca. 30-40 min), the migration distance of Marchantia sperms by their own swimming ability are estimated to be less than $3 \mathrm{~cm}$. It is indicative that sperms could not reach female gametangia solely by their own exercise capacity in the field, and therefore they must be passively transported to female gametangia before they start swimming. The swimming activity against the direction of liquid flow (rheotaxis) can be found in the behavior of human and animal sperms (Miki and Clapham, 2013). In contrast, Marchantia sperms did not show such rheotaxis under microscopic observation with a microfluidic device for sperm selection (data not shown), supporting view that the effective delivery of sperms from the male colony to the female colony are likely achieved by the flow of water. As alternative delivery pathways of bryophyte sperms to female gametangia, some transportation, such as direct transportation by microarthropods (Cronberg et al., 2006), "splash-launch" by rain drops (Brodie, 1951) and spouting out into the air (Shimomura et al., 2008), have also been reported. Because these delivery pathways supposed to be not sufficient for the invasion into the "narrow" oviduct of archegonium in archegonial receptacle of female strains, motility of Marchantia sperms might play a key role in the fertilization step, and the estimated migration distance is long enough for that purpose when they are delivered at high motile state, desirably, immediately (within 5 min) after the discharge.

Mechanisms to regulate the direction of movement and recognition of archegonium are still obscure. Human sperms direct their movement according to the gradient of certain hormones and/or chemicals (chemotaxis) and temperature (thermotaxis) in oviduct and uterine (Teves et al., 2009). Adhesiveness to the glass and aggregation in the corner of chamber suggest that mechanical guidance (mechanotaxis) may support clinging to the archegonial receptacle of female strains. Further studies using microfluidic devices to test chemotaxis and mechanotaxis may facilitate this idea.

In the present study, we analyzed the kinetic parameters of Marchantia sperm motility using a CASA system, and revealed that the migration distance of Marchantia sperms was limited to within $c a .2-3 \mathrm{~cm}$. Marchantia 

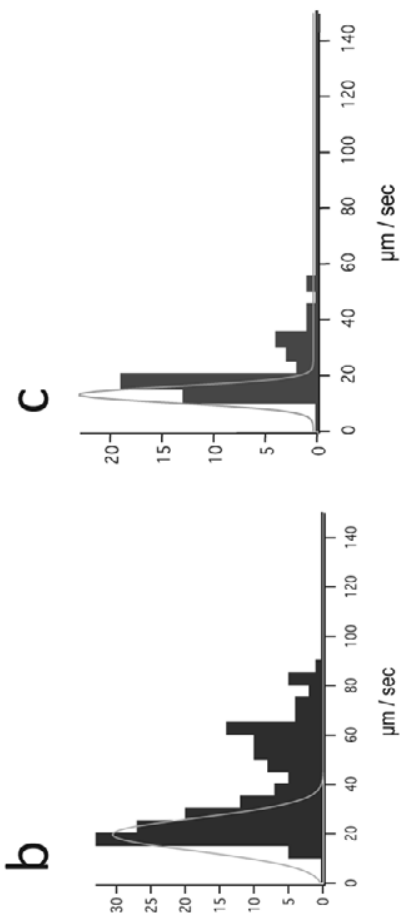

(1)

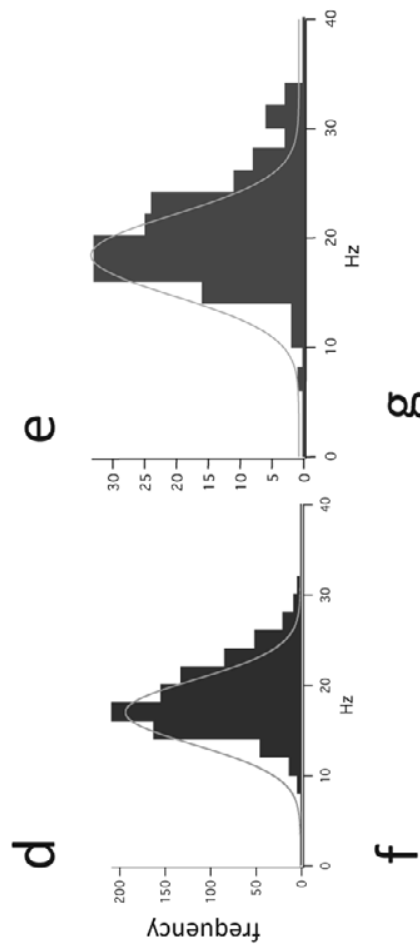

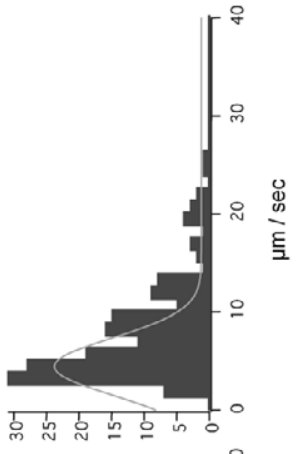
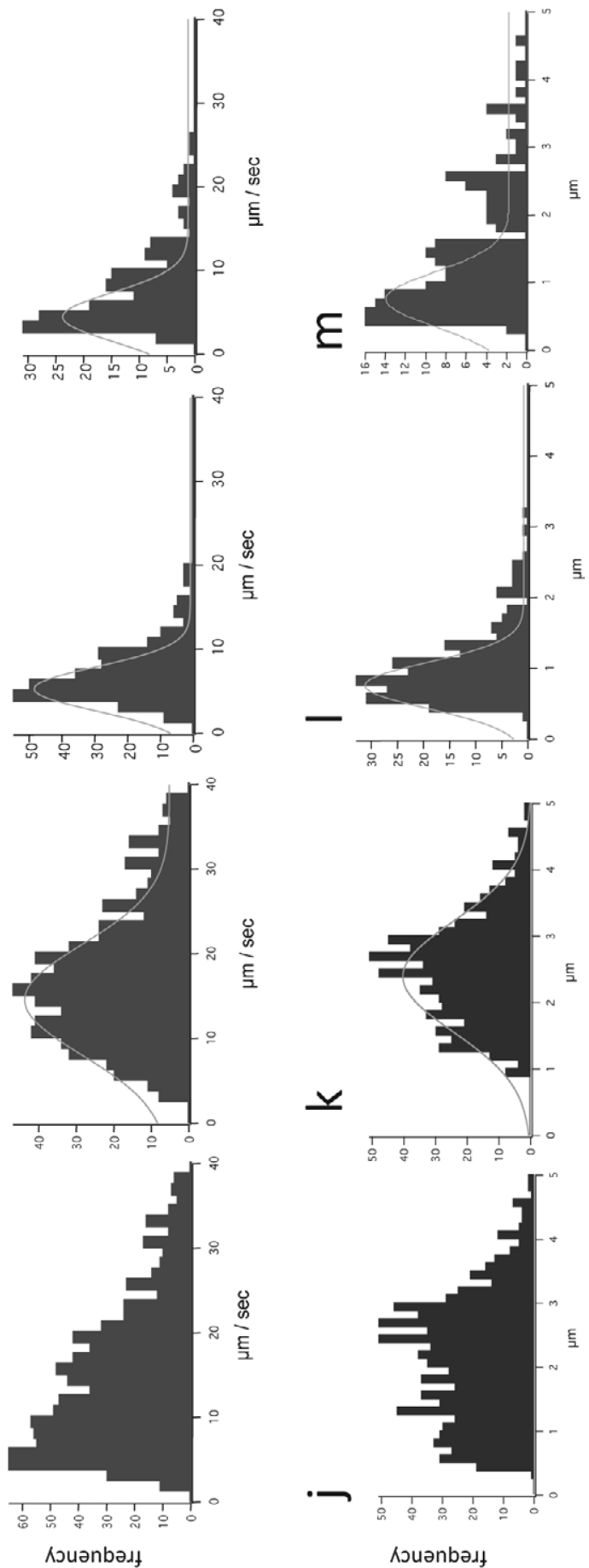

Fig. 2 Kinetic parameters of Marchantia sperm motility. a-c: curvilinear velocity, $d$ and e: frequency of lateral head place displacement, $\mathrm{f}-\mathrm{i}$ : linear (straight-line) velocity, $\mathrm{j}-\mathrm{m}$ : amplitude of lateral head place displacement. Data for $30 \mathrm{~min}$ after discharge (a, d, f, j), 60 min after discharge (b, e, i, m), and 120 min after discharge (c) are compared. According to the curvilinear velocity, sperms at 30 min after discharge were classified into two populations (with high and low motility), then their linear velocity and amplitude of head place displacement were re-analyzed: high motility group: $g$ and $\mathrm{k}$, low motility group: $\mathrm{h}$ and $\mathrm{l}$. Frequency in y-axis indicates number of sperms. Curved lines in each histogram represented the Gaussian fitting.

sperms developed their motility from non-motile to high motility states within $5^{-10} \mathrm{~min}$ after the discharge, and their motility were gradually lowered by the reduction in amplitude of lateral head displacement. Further studies to identify the underlying molecular mechanisms may important for our understanding about sperm motility and fertility in liverworts and other plant species utilizing sperms for their sexual reproduction.

\section{ACKNOWLEDGEMENTS}

We are grateful to Prof. Takayuki Kohchi (Kyoto University) for providing the male strain of Marchantia polymorpha L. (Tak-1) and invaluable suggestions, Prof. Keiji 


\section{MOTILITY OF LIVERWORT SPERMS}

Naruse for sharing SMAS. This study was partly supported by Special Coordination Funds for Promoting Sciences and Technology from the Ministry of Education, Culture, Sports, Science and Technology, Japan (to K.M.).

\section{REFERENCES}

Amann, R. P., Waberski, D. 2014. Computer-assisted sperm analysis (CASA): capabilities and potential developments. Theriogenology 81: 5-17.

Araki, T. 2012. Plant-specific transcription factor LEAFY and its possible role in gametophyte development in Marchantia polymorpha. (in Japanese) BSJ-Review 3: 147.

Brodie, H. J. 1951. The splash-cup dispersal mechanism in plants. Can. J. Bot. 29: 224-234.

Carothers, Z. B., Kreitner, G. L. 1967. Studies of spermatogenesis in the hepaticae. I. Ultrastructure of the Vierergruppe in Marchantia. J. Cell Biol. 33: 43-51.

Carothers, Z. B., Kreitner, G. L. 1968 . Studies of spermatogenesis in the hepaticae. II. Blepharoplast structure in the spermatid of Marchantia. J. Cell Biol. 36: 603-616.

Cavers, F. I. 1903. Explosive discharge of antherozoids in Fegatella conica. Ann. Bot. 17: 270-274.

Cavers, F. I. 1904. On the structure and biology of Fegatella conica. Ann. Bot. 18: 87-120, pl. 7-8.

Cronberg, N., Natcheva, R., Hedlund, K. 2006. Microarthropods mediate sperm transfer in mosses. Science 313: 1255.

Hirase, S. 1896a. Spermatozoid of Ginkgo biloba. (in Japanese) Bot. Mag., Tokyo 10: 171.

Hirase, S. 1896b. On the spermatozoid of Ginkgo. (in Japanese) Bot. Mag., Tokyo 10: 325-328.

Ikeno, S. 1896. Spermatozoiden von Cycas revoluta. (in Japanese) Bot. Mag., Tokyo 10: 367-368.

Ikeno, S. 1897. Vorläufige Mitteilung über Spermatozoiden bei Cycas revoluta. Botanisches Zentralblatt 69: 1-3.

Ikeno, S., Hirase, S. 1897. Spermatozoids in gymnosperms. Ann. Bot. 11: 344-345.

Ishizaki, K., Chiyoda, S., Yamato, K. T., Kohchi, T. 2008. Agrobacterium-mediated transformation of the haploid liverwort Marchantia polymorpha L., an emerging model for plant biology. Plant Cell Physiol. 49: 1084-1091.

Komori, K., Tsujimura, A., Ishijima, S., Tanjapatkul, P., Fujita, K., Matsuoka, Y., Takao, T., Miyagawa, Y., Takada, S.,
Okuyama, A. 2006. Comparative study of sperm motility analysis system and conventional microscopic semen analysis. Reprod. Med. Biol. 5: 195-200.

Matsuura, K., Kuroda, Y., Yamashita, K., Funahashi, H. 2011. Hydrophobic silicone elastomer chamber for recording trajectories of motile porcine sperms without adsorption. J. Reprod. Dev. 57: 163-167.

Miki, K., Clapham, D. E. 2013. Rheotaxis guides mammalian sperm. Curr. Biol. 23: 443-452.

Mirbel, C.F.B. de 1835a. Recherches anatomiques et physiologiques sur le Marchantia polymorpha, pour servir a l'histoire du tissu cellulaire, de l'épiderme et des stomates. Mém. l'Acad. Sci. l'Inst. France 13: 337-373.

Mirbel, C.F.B. de 1835b. Complément des observations sur le Marchantia polymorpha, de recherches sur les metamorphoses des utricules, et sur l'origine, developpements et la structure de l'anthère et du pollen des végétaux phanérogames. Mém. l'Acad. Sci. l'Inst. France 13: 375-436.

Renzaglia, K. S., Duff, R. J., Nickrent, D. L., Garbary, D. J. 2000. Vegetative and reproductive innovations of early land plants: implications for a unified phylogeny. Phil. Trans. Royal Soc. Lond. B 355: 769-793.

Renzaglia, K. S., Garbary, D. J. 2001. Motile gametes of land plants: Diversity, development, and evolution. Crit. Rev. Plant Sci. 20: 107-213.

Ridge, R. W., Hori, T., Miyamura, S. 1997. Analysis of flagellar movement in Ginkgo biloba sperm by high speed video microscopy. In "Ginkgo Biloba-A Global Treasurefrom Biology to Medicine", Springer-Verlag, Tokyo, pp 99107.

Shimomura, M., Yamaguchi, T., Deguchi, H. 2008. Airborne sperm of Conocephalum conicum (Conocephalaceae). J. Plant Res. 121: 69-71.

Shimomura, M. 2012. An introduction to the taxonomy and morphology of Marchantia polymorpha. BSJ-Rev. 3: 84.

Teves, M. E., Guidobaldi, H. A., Uñates, D. R., Sanchez, R., Miska, W., Publicover, S. J., Morales Garcia, A. A., Giojalas, L. C. 2009. Molecular mechanism for human sperm chemotaxis mediated by progesterone. PLoS ONE 4:e8211.

Thuret, G. 1851. Recherche sur les zoospores des algues et les anthéridies des cryptogmes. Ann. Sci. Nat. $3^{\text {me }}$ sér. 16: 5-39, pl. 1-15. 